ORIGINAL RESEARCH ARTICLE

\title{
Contraceptives: Adolescents' Knowledge, Attitudes and Practices. A Case Study of Rural Mhondoro-Ngezi District, Zimbabwe
}

\author{
Stanzia Moyo ${ }^{1 *}$ and Oswell Rusinga ${ }^{2}$ \\ University of Zimbabwe, Centre for Population Studies, Harare ${ }^{1}$; Great Zimbabwe University, Department of Sociology and \\ Social Anthropology, Masvingo ${ }^{2}$
}

*For Correspondence: Email: stanzialmoyo@yahoo.com; Phone: +263773806131

\begin{abstract}
Adolescent reproductive health behaviour has become an emerging world concern as the age at menarche and sexual debut has plummeted globally. The primary objective of the paper is to understand the importance of reproductive health education to contraceptive use among adolescents in Mhondoro-Ngezi District. The paper also investigates knowledge, attitudes, beliefs and practices (KABP) pertaining to contraceptive use among adolescents. Factors underlying KABP are further explored. In addition, this paper investigates the availability, accessibility, acceptability and affordability of contraceptives to adolescents. These issues are assessed from the perspective of all duty bearers (mothers, fathers, healthcare providers, religious leaders and secondary school teachers). The paper is based on a cross-sectional study which was conducted in Mhondoro-Ngezi area. A total of 185 adolescents aged 15-19 years were sampled with a sex ratio of 67. The study triangulated both quantitative and qualitative methods of data collection. The results in this paper indicate that adolescents in Mhondoro-Ngezi engage in early sexual debut. Despite the fact that knowledge about modern contraceptives is universal (96\%) among adolescents in Mhondoro-Ngezi, contraceptive use is very low, $21 \%$. Limited contraceptive use among adolescents in the study area is a result of the interplay of demographic, policy, socio-cultural, religious and economic factors. The study has thus recommends that the government and other relevant stakeholders to formulate policies that promote reproductive education in order to foster the utilization of contraceptives by adolescents. (Afr J Reprod Health 2017; 21[1]:49-63).
\end{abstract}

Keywords: Reproductive health problems, Contraceptives, Sex, Sexuality, Culture

\section{Résumé}

Le comportement de santé reproductive des adolescents est devenue une préoccupation mondiale émergente comme l'âge à l'apparition des règles et le premier rapport sexuel a diminué à l'échelle mondiale. L'objectif principal de ce document est de comprendre l'importance de l'éducation en matière de santé de la reproduction à l'utilisation des contraceptifs parmi les adolescents en Mhondoro-Ngezi District. Le document a également fait enquête sur les connaissances, attitudes, croyances et pratiques $(\mathrm{CACP})$ relatives à l'utilisation de la contraception chez les adolescents. Facteurs sous-jacents du CAPC sont explorées davantage. En outre, cet article examine la disponibilité, l'accessibilité, l'acceptabilité et l'accessibilité des contraceptifs à des adolescents. Ces questions sont évalués du point de vue de tous les responsables (les mères, les pères, les fournisseurs de soins de santé, des chefs religieux et des enseignants du secondaire). Ce document est fondé sur une étude transversale qui a été effectuée en zone Mhondoro-Ngezi. Un total de 185 adolescents âgés de 15 à 19 ans ont été échantillonnés avec un sex-ratio de 67. L'étude triangulés à la fois des méthodes quantitatives et qualitatives de collecte de données. Les résultats de ce travail montrent que les adolescents en Mhondoro-Ngezi s'engager dans l'activité sexuelle précoce. En dépit du fait que les connaissances sur les contraceptifs modernes est universel $(96 \%)$ chez les adolescents en Mhondoro-Ngezi, l'utilisation de la contraception est très faible, $21 \%$. Limiter l'utilisation des contraceptifs parmi les adolescents dans la zone d'étude est le résultat de l'interaction de facteurs démographiques, politiques, socio-culturelles, religieuses et économiques. L'étude a donc recommandé que le gouvernement et les autres parties prenantes pour élaborer des politiques qui favorisent l'éducation en matière de reproduction afin de favoriser l'utilisation de contraceptifs par les adolescents. (Afr J Reprod Health 2017; 21[1]:49-63).

Mots-clés: les problèmes de santé reproductive, la contraception, le sexe, la sexualité, la Culture

\section{Introduction}

Globally, there are more than 1.2 billion adolescents between the ages of 10 and 19 years and nine in 10 of them live in developing countries ${ }^{1}$. In Zimbabwe, adolescents number about 3015 820, representing over $35 \%$ of the total population ${ }^{2}$. In addition, $67 \%$ of these adolescents live in rural areas ${ }^{3}$. However, in 
many parts of the world, adolescents have been a neglected group largely because of cultural sensitivities and gender disparities regarding sexuality ${ }^{4}$.

Evidence has shown that reproductive health problems among adolescents in Zimbabwe continue to increase unabatedly irrespective of the fact that national HIV infection rates have declined from about $34 \%$ in 1995 to $13.7 \%$ in $2009^{5}$. Adolescents are faced by a myriad of reproductive health problems. These include early sexual debut, unwanted pregnancies, unsafe abortions, sexually transmitted infections (STIs), HIV and AIDS and early marriages ${ }^{6}$. It should be noted that unsafe sex is the key risk factor for poor reproductive health among adolescents ${ }^{7}$.

Premarital sexual activity is common among adolescents in Zimbabwe, ranging between $29 \%$ and $52 \%^{8-10}$. It is a key factor undergirding adolescent reproductive health issues. About $55 \%$ of the overall pregnancies among adolescents aged 14 to 19 in Zimbabwe were reported as unwanted ${ }^{11}$. Of great concern is the fact that unwanted pregnancies among adolescents in most cases results in induced abortions normally performed under unhygienic and clandestine conditions. About $7 \%$ of all pregnancies to Zimbabwean teenage girls end up in unsafe abortions ${ }^{11}$. It has been noted that $50 \%$ of all HIV infections occur during adolescence and young adulthood $^{5}$. Moreover, $42 \%$ of antenatal clinic attendees, less than 20 years old, tested HIV positive in Harare $^{11}$. HIV and AIDS prevalence among adolescents increased by $45 \%$ from 2005 to $2006^{12}$, 13

\section{Factors influencing reproductive health problems among adolescents in Zimbabwe}

Socio-cultural and economic contexts facilitate adolescents to engage in risky sexual behavior which put them at risk of unwanted pregnancies and contracting HIV and other sexually transmitted infections. Girls in particular are increasingly vulnerable to the approaches of elderly men who are seeking 'HIV free' partners and are able to exploit the economic marginality of young women and girls by offering them gifts and money for $\operatorname{sex}^{14}$.

In addition, adolescents in general have limited information on reproductive health. Even though adolescents and adults alike, report that adolescents do engage in sexual activity, they are against the idea of making reproductive health services accessible to adolescents ${ }^{9}$. This attitude emanates from the cultural norm that adolescents must not engage in sexual activity before marriage, especially girls who are expected to marry as virgins $^{11}$.

There is a conspiracy of silence regarding sexuality in Zimbabwe ${ }^{15}$. Consequently, the subject of adolescent sexuality remains a taboo. While parents are perceived to be the logical source of information, they often do not discuss sexuality issues with their children because culturally, sexual education is the exclusive domain of aunts and uncles. Moreover, traditional mechanisms of sex education have not only weakened in recent decades, but are focused on gendered sexual socialization and not on the promotion of sexual health ${ }^{14}$.

Furthermore, there is still no policy or law that specifically caters for the reproductive health needs of adolescents in Zimbabwe. Consequently, this has made it difficult to develop curricula which include a component of adolescent contraceptive use during nurses' and doctors' training. This has also made it difficult for the Ministry of Education to develop comprehensive national adolescent reproductive health syllabi for the respective grades. The current AIDS Education which is being taught from Grade 4 up to Form 6 covers all the topics relating to family life but does not address sensitive issues such as contraception, sex and sexuality.

Historically, many studies implicitly conceptualized risky sexual behavior as an individual-level phenomenon. However, emerging evidence indicates that a spectrum of contextual factors are prominent and interact with each other to promote risky sexual behavior, including psychological, social, relational, familial, developmental, structural, environmental and cultural factors ${ }^{16}$. In other words, the limited use of contraceptives among adolescents should be viewed within the prism of interacting contextual factors. This is precisely because sex and sexual behavior are essentially socio-cultural ${ }^{17}$. While a number of studies have been conducted on KABP in reproductive health among adolescents, no studies have investigated the levels of contraceptive use and factors undergirding such levels specifically for 
adolescents in rural Mhondoro-Ngezi. No particular reference has been made on assessing the significance of reproductive health education as well as its implications on contraceptive use among adolescents in Mhondoro-Ngezi. This is however essential in order to influence and support behaviour change aimed at sensitizing community about sexual and reproductive health needs of adolescents. Furthermore, reproductive health has become multidimensional; its policies and interventions now extend beyond health per se $e^{7}$.

\section{Objectives}

The broad objective of the study was to establish the importance of reproductive health education to contraceptive use among adolescents in MhondoroNgezi District. The study specifically sought to:

1. identify the levels of contraceptive use among adolescents in Mhondoro-Ngezi;

2. examine the factors under girding contraceptive use among adolescents;

3. investigate knowledge, attitudes, beliefs and practices pertaining to use of contraceptives among adolescents; and

4. assess the availability, acceptability, accessibility and affordability of reproductive health services to adolescents.

\section{Methods}

\section{Study design}

The study was cross-sectional survey targeting males and females aged 15 to 19 years mainly because it is the population's window of hope. The study triangulated both qualitative and quantitative methods. Focus group discussions (FGDs) and key informant interviews were used for the qualitative part of the study, while a survey was used for collecting quantitative data.

\section{Data collection and tools}

\section{Survey}

A total of 185 questionnaires were administered to adolescents aged 15 to 19 years. This was meant to quantify the magnitude of contraceptive use among adolescents in the study area. Knowledge, attitudes, beliefs and practices pertaining to contraceptive use among adolescents were also quantified using a survey.

\section{Sample size determination}

To determine the number of respondents in the survey, the following formula was used: $\mathrm{N}=$ $\left(\mathrm{z}^{2 *} \mathrm{p}^{*} \mathrm{q}\right) / \mathrm{e}^{2}$; Where: $\mathrm{N}=$ desired sample size; $\mathrm{z}^{2}=$ the standard normal deviate set at 1.96 which correspond with $95 \%$ confidence intervals; $p=$ the proportion in the target population estimated to be aged 15 to $19 ; \mathrm{q}=$ the estimated proportion of the target population who are not adolescents (1-p); $\mathrm{e}^{2}=$ desired level of precision and in this study is set at 0.05 . According to the 2002 census, the total population was estimated at 4726 in MhondoroNgezi and 648 were adolescents. Therefore, the following calculations were used to obtain the sample size $\left(1.96^{2} * 0.14 * 0.86\right) / 0.05^{2}=185$.

\section{Sampling procedure}

Respondents were selected from all the ten Enumeration Areas (EAs) as demarcated by the CSO Master Sample of 2002. A probability to proportional size $\left(\mathrm{N}_{\mathrm{h}} / \mathrm{N}^{*} \mathrm{n}\right)$ was used to determine the number of respondents from each EA. Where: $\mathrm{N}_{\mathrm{h}}=$ number of adolescents per given EA; $\mathrm{N}=$ total number of adolescents in all the ten EAs, and $n=$ sample size. Given that the total number of adolescents in EA1 is 111 and total adolescents in Mhondoro-Ngezi is 648, the calculation of adolescents to be selected in EA1 is $111 / 648 * 185$ $=32$. The rest of the respondents who were selected per each EA are shown in Table 1.

The household list used in the 2002 census was used to identify the number of households per EA. A systematic sampling method was used to select the number of households in each EA. After a household had been selected the eligible respondent was identified by enumerating all potential respondents in the household. If in the household there were more than one adolescent, each adolescent was assigned a number and then a random procedure was conducted to select one adolescent.

\section{Focus group discussions}

Five FGDs (using a FGD guide) were carried out in 
Table 1:Distribution of Number of Adolescents, Sampled Adolescents and Number of Households per EA in Mhondoro-Ngezi (Ward 5)

\begin{tabular}{llll}
\hline $\begin{array}{l}\text { EA } \\
\text { Code }\end{array}$ & $\begin{array}{l}\text { Number of } \\
\text { Adolescents }\end{array}$ & $\begin{array}{l}\text { Sampled } \\
\text { Adolescents } \\
\text { from each EA }\end{array}$ & $\begin{array}{l}\text { Number } \\
\text { Households }\end{array}$ \\
\hline 1 & 111 & 32 & 162 \\
2 & 54 & 15 & 97 \\
3 & 56 & 16 & 95 \\
4 & 51 & 15 & 72 \\
5 & 55 & 16 & 88 \\
6 & 39 & 11 & 68 \\
7 & 54 & 15 & 109 \\
8 & 101 & 29 & 103 \\
9 & 61 & 17 & 131 \\
10 & 66 & 19 & 12 \\
Total & $\mathbf{6 4 8}$ & $\mathbf{1 8 5}$ & $\mathbf{9 3 7}$ \\
\hline
\end{tabular}

this study. However, separate FGDs were conducted with adolescent boys, girls, women and men above 30 years and one FGD combined male and adolescent females. Norms and values undergirding contraceptive use among adolescents were also clarified by FGDs. Furthermore, FGDs also helped to obtain normative information about participants' knowledge, attitudes, beliefs and practices pertaining to contraceptive use among adolescents.

\section{Key informant interviews (KII)}

A total of 21 key informant interviews (using a key informant interview guide) were undertaken with healthcare providers, religious and traditional leaders and school teachers. Eight healthcare providers were randomly selected from the two health centers in the area. In addition, three religious leaders, two traditional leaders, and eight secondary school teachers were also randomly selected. Interviews helped to establish the levels of contraceptive use among adolescents and the factors undergirding such levels. They also helped establish the KABP of key informants with regards to contraceptive use among adolescents.

\section{Data analysis}

Survey data was analyzed using the Statistical Package for Social Scientist version 16.0. The package facilitated the analysis of data through frequency distributions and cross tabulations. D.T
Search was used to analyze qualitative data using the thematic approach.

\section{Ethical considerations}

The study respected freedom to participation. Participants voluntarily consented to participate without coercion. There was no deception when informed consent was emphasized. Participants were told about the following: the purpose of the research; what was expected of a research participant (including the amount of time likely to be required for participation); expected risks and benefits (including psychological and social); the fact that participation was voluntary and that one can withdraw at any time with no negative repercussions; how confidentiality was to be protected; the name and contact information of the local investigator to be contacted for questions or problems related to the research; and the name and contact information of an appropriate person to contact with questions about one's rights as a research participant. The study adhered to research principles pertaining to privacy and confidentiality.

\section{Results}

\section{Socio-demographic characteristics of respondents}

Table 2 presents the socio-demographic characteristics of respondents. More females (52\%) than males (48\%) participated in this study. The mean age of respondents was 17 years. The majority of the respondents, $48 \%$, were in the 16-17 years age group, $17 \%$ of adolescents were in the 14 -15 years age group while $35 \%$ was in the $18-19$ years age group. Ninety-six were never married, $3 \%$ were married while only $1 \%$ of the adolescents were divorced/separated. The majority of the respondents $(80 \%)$ reported that they were school pupils, $8 \%$ were farmers and $7 \%$ were vendors. The mean years completed at school by respondents is 10 years. The minimum years completed is three years while the maximum is 13 years. A higher proportion of the respondents $(80 \%)$ reported that they had secondary education while close to $1 \%$ had primary education. 


\section{Sexual experience of adolescents}

reported a higher percentage $(43 \%)$ than females (33\%) of ever had sex. It was equally important to
Sexual activity is common among adolescents. Thirty-eight percent of respondents agreed ever had sex (Table 3). It was important to note that males note that in the age group 16-17, both males and

Table 2: Percentage Distribution of the Respondents by Selected Socio-Demographic Characteristics

\begin{tabular}{lccc}
\hline Demographic Characteristics & $\begin{array}{l}\text { Male } \\
\text { Number (\%) }\end{array}$ & $\begin{array}{l}\text { Female } \\
\text { Number (\%) }\end{array}$ & $\begin{array}{c}\text { Total } \\
\text { Number (\%) }\end{array}$ \\
\hline Age & & & \\
$14-15$ & 13.2 & 22.0 & 17.7 \\
$16-17$ & 47.0 & 48.4 & 47.5 \\
18-19 & 39.8 & 29.7 & 34.8 \\
Total & $\mathbf{1 0 0 . 0}$ & $\mathbf{1 0 0 . 0}$ & $\mathbf{1 0 0 . 0}$ \\
Marital Status & & & \\
Never married & 96.2 & 94.6 & 96.0 \\
Married & 2.8 & 3.3 & 2.8 \\
Divorced/Separated & $\ldots$. & 2.1 & 1.2 \\
Total & $\mathbf{1 0 0 . 0}$ & $\mathbf{1 0 0 . 0}$ & $\mathbf{1 0 0 . 0}$ \\
Occupation & & & \\
Student & 77.2 & 81.8 & 79.7 \\
Farmer & 13.0 & 3.9 & 8.1 \\
Self-employed (vendor, dress maker etc.) & 6.2 & 8.5 & 7.5 \\
Formally Employed (teacher, nurse etc.) & 1.0 & 2.0 & 1.5 \\
Domestic worker & 0.1 & 2.3 & 1.2 \\
Unemployed & 2.5 & 1.5 & 2.0 \\
Total & $\mathbf{1 0 0 . 0}$ & $\mathbf{1 0 0 . 0}$ & $\mathbf{1 0 0 . 0}$ \\
Level of Education & & & \\
Primary & 1.0 & 1.8 & 1.4 \\
Secondary & 80.6 & 79.2 & 79.9 \\
Tertiary & 18.4 & 19.0 & 18.7 \\
Total & $\mathbf{1 0 0 . 0}$ & $\mathbf{1 0 0 . 0}$ & $\mathbf{1 0 0 . 0}$ \\
\hline N & & &
\end{tabular}

females had a higher percentage of friends having sex. Girls in the 16-17 age group recorded a higher percentage (66\%) than boys (56\%). Reasons such as fun, pleasure, coercion by love partners, married, peer pressure, sign of love, financial support, gain experience and boredom were cited in all FGDs and KII.

Table 3: Percentage Distribution of Adolescents Sexual Experience by Age and Sex

\begin{tabular}{|c|c|c|c|c|}
\hline \multirow[b]{2}{*}{ Sex } & \multicolumn{4}{|c|}{ Ever had Sexual Intercourse } \\
\hline & & Yes & No & Total \\
\hline \multirow[t]{5}{*}{ Males } & $\begin{array}{l}\text { Age } \\
\text { group }\end{array}$ & $\begin{array}{l}\text { Number } \\
(\%)\end{array}$ & $\begin{array}{l}\text { Number } \\
(\%)\end{array}$ & $\begin{array}{l}\text { Number } \\
(\%)\end{array}$ \\
\hline & $14-15$ & 2.0 & 98.0 & 100 \\
\hline & $16-17$ & 56.9 & 43.1 & 100 \\
\hline & $18-19$ & 41.2 & 58.8 & 100 \\
\hline & Total & 43.2 & 56.8 & 100 \\
\hline \multirow[t]{4}{*}{ Females } & $14-15$ & 16.7 & 83.3 & 100 \\
\hline & $16-17$ & 65.8 & 34.2 & 100 \\
\hline & $18-19$ & 26.3 & 73.7 & 100 \\
\hline & Total & 32.6 & 67.4 & 100 \\
\hline Both & Total & 37.7 & 62.3 & 100 \\
\hline
\end{tabular}

$\mathrm{N}=185$

Knowledge about contraceptive is universal, $96 \%$, with no variation by sex. However, it is interesting to note that the universal knowledge about contraceptives does not transcend to use. Contraceptive use among adolescents in this study is very low. Of the $37 \%$ adolescents who had ever had sexual intercourse, $79 \%$ had not ever used any contraception at all. In addition, for the $21 \%$ who ever used contraception only $25 \%$ reported current use. It was also interesting to note that females in all cases had a higher percentage $(82 \%)$ of noncontraception use than males (18\%).

Asked about the type of contraception sexually active adolescents used, all male adolescents mentioned the condom (modern method). No one ever mentioned traditional methods like withdrawal and folk methods. It was interesting to note that the majority $(95 \%)$ of females who ever used contraception stated that they used the male condom, $3 \%$ pills, $1 \%$ injectables 
and female condoms. None ever used IUD or implant.

Peers seem to be the source of contraception for sexually active adolescents. Of the 25\% adolescents who are currently using contraception, it was interesting to note that the majority $(80 \%)$ are getting them from their friends, whilst $12 \%$ and $5 \%$ are getting them from shops and beer outlets respectively. Two percent and 1\% of respondents are getting contraceptives from community health care distributors and relatives respectively. It was surprising to note that clinics are only having $0.3 \%$ of adolescents accessing contraceptives.

\section{Knowledge about contraceptives}

Results seem to reveal that knowledge about natural methods of contraception is very limited among adolescents. The majority of respondents (82\%) reported that they do not know any natural method of family planning. Percentage distribution by sex revealed that there was no major variation by sex on knowledge about natural methods of contraception ( $80 \%$ males and $83 \%$ females).

Breastfeeding seem to be the most known method of family planning known by adolescents. Of the $18 \%$ respondents who know any natural method of family planning $89 \%$ mentioned breastfeeding whilst $11 \%$ stated withdrawal. It was interesting to note that none of the respondents mentioned periodic abstinence as a method of natural family planning.

Knowledge about modern contraception seems to be universal among adolescents. The majority of respondents, $98 \%$, stated that they had ever heard about modern methods of contraception. There was no marked difference of knowledge by sex (99\% males and $97 \%$ females).

Condoms seem to be the most known method of family planning among adolescents. Of the $98 \%$ respondents who had ever heard about modern methods of family planning, $84 \%$ had heard about condoms, $11 \%$ about pills, $2 \%$ about implants and $3 \%$ about injectables. It was interesting to note that respondents displayed profound ignorance about female and male sterilization and also IUD.

All adolescents in FGDs stated that they were more aware of condoms than other methods of contraception since most of the adverts they see and hear (both in print and electronic media) are about condoms. They also highlighted that they know bits and pieces of pills, implants and injectables from biology lessons at school. Adolescents highlighted that lack of knowledge about other forms of contraception was primarily due to the fact that parents, religious and traditional leaders and healthcare providers do not teach them about contraceptive use. Adolescent stated that lessons about sex and sexuality were considered a taboo in their community. As one adolescent stated in FGD:

Lessons about contraceptive use among
adolescents are a taboo in our community.
Parents and leaders believe that if we are
taught about contraception, that's a green
light to practice pre-marital sex and
prostitution. It was interesting to note that all parents, religious and traditional leaders were against the issue of teaching adolescents about contraceptive use. They argued that teaching adolescents about contraception is one way of encouraging early sexual debut and prostitution. One male parent in a FGD was totally against the teaching of adolescents about contraception as he fumed:

Should I find my wife teaching my daughter about contraception that will be the end of our marriage? I will kill her and the daughter. I do not entertain prostitutes at my home. My culture does not entertain such nonsense.

In interviews, $90 \%$ of teachers revealed that they only mention the words contraceptives, condoms, pills, depo-provera, IUD male and female sterilization as a call of duty and only when teaching biology. They also emphasized the fact that there is no National Adolescent Reproductive Health policy where educationist can draw a national syllabus for the subject. One female secondary school teacher stated in an interview that they teach issues of reproductive health to adolescents in Guidance and Counseling ( $\mathrm{G}$ and $\mathrm{C}$ ) lessons. However adolescents in all FGDs argued that issues of reproductive health and contraceptive use in particular, are never mentioned at all in $\mathrm{G}$ and $\mathrm{C}$ lessons. One female adolescent in a FGD argued that: 
$G$ and $C$ only cover topics ranging from bathing, washing and the general maintenance of a clean environment. More so, it is only taught once a week (40 minutes only). In addition, it is not an examinable subject, as such, even our teachers do not consider it seriously.

Religious leaders in this study do not teach adolescents about contraceptives. A higher proportion of the respondents $(87 \%)$ reported that churches do not talk about contraceptive use (Figure $1)$.

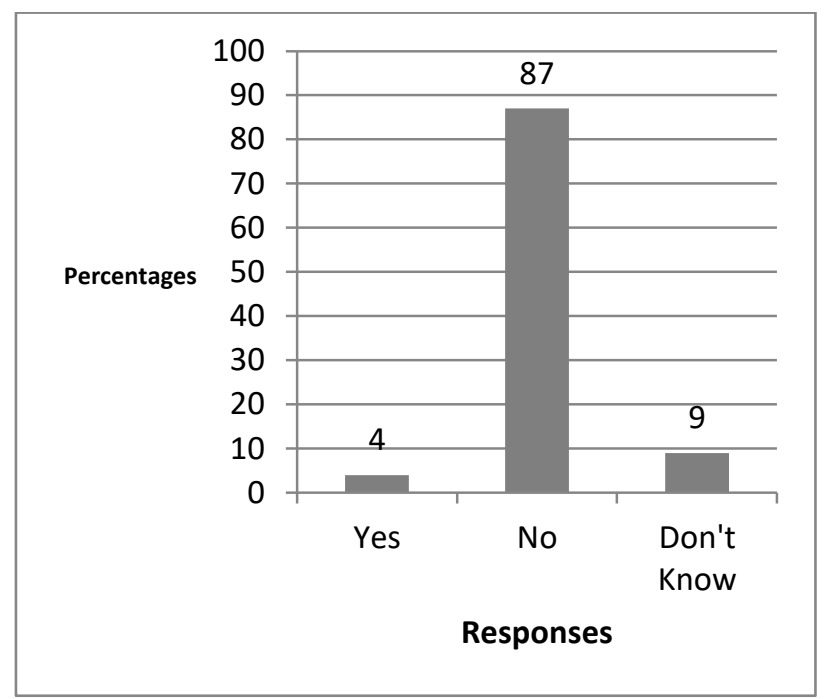

Figure 1: Percentage Distribution on Whether Churches Talk About Contraceptives to Adolescents $(\mathrm{N}=185)$

In all adolescents FGDs, respondents stated that churches do not talk about contraceptive use among adolescents. They argued that church leaders feel that it is ungodly and contrary to abstinence and faithfulness taught in the Bible. It was interesting to note that these comments were similar to the ones echoed by a religious leader in a KII. The pastor stated that:

In my weekly preaching, I always emphasize on abstinence rather than contraceptive use among unmarried adolescents and youths. I strongly feel that if $I$ am to teach contraceptive use to adolescents, I will be encouraging adultery and infidelity which are all sins before God.
KII with healthcare providers revealed that they are mandated to provide information, education and communication (IEC) on reproductive health to adolescents. The nurses said they provide IEC to adolescents particularly on public gatherings. However, adolescent in all FGDs argued that healthcare providers do not teach them about reproductive health and contraceptive use at any place. They argued that they only see nurses mostly at sports days where they will be primarily providing first aid.

Adolescents seem to be advising each other on contraceptive use. Results revealed that the majority $(42 \%)$ of respondents stated that their peers advise them about contraceptives (Table 4). It was also surprising to note people who interact with adolescents on day to day basis like teachers, health workers, religious leaders and parents provided very little contribution to when it comes to advising adolescents on sexuality issues (Table 4). This means that adolescents depend on advises from their misinformed friends and their own personal experiments.

There were contradictions from key informants on who is providing knowledge about contraceptive to adolescents. KII with religious and traditional leaders revealed a perception that parents, aunts, uncles, teachers and health care providers are advising adolescents on sexuality issues. On the other hand, interviews with teachers revealed the perception that parents, aunts, uncles, grandparents and healthcare providers (HCP) are advising adolescents on sexuality issues. However, interviews with HCPs revealed a perception that

Table 4: Percentage Distribution of Who is Advising Adolescents on Contraceptive Use and Sexual Issues

\begin{tabular}{ll}
\hline $\begin{array}{c}\text { Who should advice adolescents } \\
\text { on sexual issues? }\end{array}$ & Number (\%) \\
\hline Parents & 2.1 \\
Aunt/uncle & 2.2 \\
Grandparents & 4.0 \\
Peers & 42.4 \\
Health workers & 1.0 \\
Teachers & 2.1 \\
Other siblings & 14.6 \\
Religious and traditional leaders & 2.9 \\
Media & 8.0 \\
None & 21.0 \\
Total & $\mathbf{1 0 0}$ \\
\hline
\end{tabular}

$\mathrm{N}=185$ 
teachers, parents, aunts, uncles and grandparents are advising adolescents. In all FGDs of males and females above thirty-five years (representing parents) they stated teachers, HCPs, religious and traditional leaders as people advising adolescents. However, in all adolescent FGDs, they revealed that peers and other siblings advise them on contraception issues whilst others do not even have anyone to advise them. It was also interesting to note that adolescents highlighted the absence of parents, aunts, uncles, teachers, and grandparents, religious and traditional leaders in this crucial process of socialization in their lives.

Adolescents are of the view that they need to be advised on issues on contraceptive use. Survey results indicated that the majority of respondents (40\%) stated that parents should advise them on sexual issues, while $27 \%$ and $14 \%$ respectively mentioned aunts/uncles and health workers. Grandparents, peers, other siblings, traditional leaders and religious leaders were mentioned by $6 \%$, $4 \%, 4 \%, 3 \%$ and $2 \%$ of respondents respectively.

However, in FGDs with adolescents, they highlighted that dissemination of knowledge about contraceptives is a fundamental responsibility of all duty bearers. All KIIs also revealed the perception that a holistic approach should be used in the dissemination of correct information about the use of contraceptives to adolescents.

\section{Knowledge of availability of contraceptives to adolescents}

Male condoms are the widely known form of contraception available to adolescents. The majority of respondents (89\%) stated that condoms are always available. In a multiple response question, $90 \%, 89 \%, 94 \%$ and $82 \%$ of respondents stated that condoms are available in shops, beer outlets, and clinics and from the community health distributers respectively. However, in clinics, healthcare providers highlighted that they do not carry out female and male sterilization. Cases of sterilization were said to be referred to St Michael's hospital (a referral hospital in the area).

The majority of adolescents (78\%) were unaware of the availability of female contraceptives at clinics. Whilst health care providers stated in KII that female contraceptives such as pills, injectables, implants and IUD were also available at health centres, it was interesting to note that most adolescent clients were not aware of such facilities. Adolescents argued that duty bearers have not told them about such a provision. To substantiate adolescents' assertion, one adolescent in a female FGD stated that:

We do not know about the availability of female contraceptives at our local clinics. Nurses have never told us about this before. Actually whenever you visit the clinics, the old aged nurses will always look busy to explain anything to you. We are also even afraid to ask any question pertaining to contraception.

\section{Accessibility of contraceptives to adolescents}

Adolescents seem to be having problems in accessing contraceptives. Resultantly, this has contributed to non-use of contraceptives. Of the $37 \%$ adolescents who are sexually active $64 \%$ said they are not using contraceptives. Female adolescents in FGDs argued that they do not use contraception because of societal norms and values which link premarital sex to prostitution. One female adolescent echoed this in a FGD:

\section{Society disapproves pre-marital sex. Resultantly accessing contraceptives especially going to the clinic constitutes a public admission of having had sex. This is also automatically linked to promiscuous behavior.}

It should be noted that this social disapproval of premarital sex has limited especially adolescent girls' knowledge of and access to health services. Consequently, adolescent girls are left with non- use of contraception as the only option.

Female adolescents in a FGD also stated that they cannot easily access contraceptives from health services because they are not married and are under the age of 18. One female in adolescent FGD argued that:

Contraceptives (especially pills, IUD, implants, female condoms and injectables) are only accessed by married and 
adolescents above 18 years at the clinic. It seems it is a crime for an adolescent below 18 years to be seen with contraceptives. It is very astonishing that health care providers fail to recognize that we are also sexually active and in need of contraceptives.

It was interesting to note that healthcare providers only give contraception (especially female types) to married and those adolescents above 18 years. All healthcare providers in KII concurred with the aforementioned assertion. One of the healthcare providers clearly echoed that:

My professional practicing guidelines do not allow me to provide contraception to anyone under the age of 18. In fact the Legal Age of Majority Act describes anyone under 18 years as a minor. I can only give contraception to an adolescent below 18 years provided the she brings any one of her parents.

The issue of bringing a parent in order to access contraception proved a bitter pill for adolescents to swallow in FGDs. Adolescents in all FGDs preferred their reproductive health issues to be treated without most confidentiality.

Even though contraception is available at health centres, adolescents would not feel free to get contraceptives at a health centre. The majority of respondents, $79 \%$, stated that they would not feel free to get contraceptives at a health centre. It was interesting to note that analysis by sex revealed that more females, $88 \%$, than males, $59 \%$, would not feel free to get contraceptives at health centres. This could be largely due to differences in how society views premarital sex for boy and girls. The double standards of culture as noted in female adolescent FGD has emphasized virginity to females whilst males are allowed to graze around before marriage.

In all adolescent FGDs, 90\% of the participants argued that they do not feel free to get contraceptives from health centres because of the stigma attached to sexually active adolescents by HCP. In addition, hostility, old age and judgmental attitude of HCP encumber the utilization of health care services by adolescents. One female adolescent in a FGD stated that health-care providers at clinics have ages similar to their parents. As such, they do not feel free to ask anything about contraception because of the cultural register which should exist between parents and children. Female adolescents in the FGD also argued that health care providers lack basic principles of confidentiality which is fundamental in their execution of duties. The female adolescent echoed that:

Nurses will just tell anyone that you took some condoms or asked for female contraception at the clinic. If you take any contraception at the clinic, you should know that before sunset everyone (including your parents and church members) in the community will be aware that you have taken some contraceptives at the clinic. This will be definitely the hot selling news of the day. The news will spread like a veld fire accompanied by a might wind.

It was also interesting to note that whilst condoms were available at the clinics, all adolescents expressed concern on the distribution points of condoms. All adolescents in FGDs asserted that condoms are placed in a card-board box at the recording desk, consultation and treatment rooms. The aforementioned assertion was also supported by all healthcare providers in KII. One of the healthcare providers in a KII stated that:

We have condoms throughout the clinic. We have condom boxes for our patients at the temperature and weight recording desk, consultation and treatment rooms. There are plenty of condoms I tell you.

However, all adolescents in FGDs were bitter about the accessibility of condoms at the aforementioned points at clinics. Adolescents in FGDs argued that condom collection points at the clinics will be full to capacity with patients including to a very large extent old ages. One female adolescent in an FGD argued that:

You will not dare make a mistake of tempering with condoms packets at the clinic because all the patients, including the old aged, will be looking at you. Before reaching home, your parents will have been told the 'shocking news'. 
Another female adolescent had this to say in an FGD:

How can you take a condom from the collection points at the clinics when all patients (some friends, relatives, and teachers, religious and traditional leaders) in all age groups will be staring at you?

While condoms are available in shopping centres, female adolescents in a FGD argued that they were not using contraceptive especially condoms because of the stigma attached by society to sexually active adolescent and lack of privacy. One female adolescent had this to say in a FGD:

Society thinks that any one (especially female) who buys condoms is a prostitute. Even the shop-keeper will ask you why you are buying the condoms. Furthermore, there is no privacy in shops. In most cases the shops will be having other customers. I would need privacy when buying condoms. However if there are other customers, the whole concept of privacy diminishes.

Thus, while condoms are available at clinics and shops, it is the issue of privacy and confidentiality which is hindering the accessibility of such contraceptives. Resultantly most of the sexually active adolescents would not use them.

\section{Attitudes and acceptability of contraception by adolescents}

Sex without condoms seems to be widely accepted by adolescents. The majority of the respondents $(77 \%)$ stated that they would have sex without condoms. Analysis by sex revealed that more males (94\%) than females $(60 \%)$ reported that they would have sex without condoms. As long as adolescents widely accept sex without condoms, it therefore means most of them will not use condoms.

Male adolescents who would not use condoms argued in a FGD that condoms are emasculate. In addition, males argued that they would not use condoms so as to enjoy the natural feeling of sex.

Adolescents seem to have negative perceptions about condoms. The majority of respondents $(61 \%)$, stated that condoms have small holes that allow HIV and STI infection to pass through, $21 \%$ disputed whilst $19 \%$ do not know. Male adolescents (79\%) are more likely to believe that condoms have small holes that allow HIV and STI infection to pass through than females (43\%).

In FGD of adolescent boys, most of them were bitter about condoms especially the blue ones provided at health centres. One boy in a FGD remarked:

Blue condoms found at health centres have holes. Water licks from these condoms should you fill them. Under such a scenario, you do not need a rocket scientist to tell you that you are not protected from risks of unwanted pregnancies, STI and HIV infection.

Adolescents feel that condoms have negative effects on sexual intercourse. The majority of the respondents, $86 \%$, stated that condoms defeat the thrill and objective of sex whilst $12 \%$ disputed and $3 \%$ do not know. Males have a higher percentage (90\%) of concurrence as compared to females (82\%). Resultantly, this possibly explains the high risks to unwanted pregnancies, STI and HIV infection, early marriages and abortions.

As a result of the negative perceptions about condoms, adolescents in this study seem to be reluctant to purchase condom if they do not have them. The majority of respondents, 69\%, reported that they would not buy condoms even when they do not have. Analysis by sex revealed that the majority of females, $78 \%$, stated that they would not buy condoms if they do not have them as compared to $65 \%$ males.

Adolescents who would by condoms seem to be aware of the negative effects of unprotected sex. Respondents, $31 \%$, who stated that they would buy condoms argued that condoms protect them from risks of early and unwanted pregnancies (which have ripple effects of early marriages, school dropout, and abortion), STIs and HIV and AIDS.

Adolescents also have negative attitudes towards contraceptive pills. About $86 \%$ of respondents revealed that contraceptive pills cause infertility. In addition, $78 \%$ of respondents stated that family planning causes deformed babies. More 
so, $72 \%$ of respondents also believed that female contraceptives cause menstrual disruptions. This also seems to reveal the fact that adolescents are ill informed about the effects of contraceptive pill. This should not be surprising considering the fact that they get most of reproductive health information from their friends. However, such negative perceptions about family planning pills greatly influence non-use of contraception among adolescents.

Acceptability of contraceptives by adolescents seems to be worsened by the disapproval stance taken by parents. In a FGD of female adolescents one girl remarked:

It is very difficult to use contraceptives because I do not have anywhere to hide them. I will be in hot soup should my mother discover contraceptives in my bedroom. So why should I use things which I know will cause serious problems for me.

One female adolescent in the same FGD also stated that because of parents' disapproval she finds it better to ask for a contraceptive a pill (which she will take once before or just after sex) from a friend above 20 years whenever she thinks she will have sex. This clearly demonstrates utter ignorance on how contraceptive pill should be taken.

In a FGD with females over 30 years (parents) one parent was very bitter about the provision of contraceptives to adolescents especially girls. She bitterly echoed that:

Why give adolescent girl contraception when she does not have a husband? Why family planning to adolescents when they are not married? I need contraception because I have a husband. This must not be a goat situation where everyone has a beard. Should I find my adolescent daughter with any form of contraception, I will definitely kill her.

The aforementioned comment partly explains why some adolescent girls will not use any contraception. More so, it also explains the ignorance in some parents on contraception and adolescents sexuality.
However, it was interesting to note the double standards of culture when it comes to contraceptive use among adolescent. While parents would fume about contraceptive use among female adolescents, male pre-marital sex and male condom use were viewed as signs of manhood. In a male parent FGD, one of them echoed that:

It's just normal in our culture for a man to have pre-marital sex. I will not be angry if I see my adolescent son having condoms. This means that he is grazing around before marriage which is culturally approved of men. More so, remember manhood in our culture, is shown by the number of women you would have 'conquered'.

The above mentioned views partly explain why male adolescents had a higher percentage of accessing condoms (93\%) than $12 \%$ of their female counterparts

\section{Affordability of contraception}

Most adolescents seem to be able to buy the male condoms. The majority of adolescents $(97 \%)$ stated that male condoms are affordable since they just cost one rand for a packet of three. However, female contraceptives such as the pill, implants, IUD and the injectables seem to be beyond the reach of most adolescents. The majority of adolescents (89\%) stated that they are not able to pay for female contraceptives should they need them. Female adolescents argued in FGD that the facilities of female pills, depo and implant provided at local clinics require a yearly subscription card of US\$20. Such an amount has been argued by adolescents to be beyond their reach since they are school pupils.

\section{Discussion}

Most of the sexually active adolescents in this study do not use contraception. Seventy-nine percent of the $37 \%$ sexually active adolescents confirmed the afforested. This percentage (79\%) is lower than $90.3 \%$ reported in 2005-6 $\mathrm{ZDHS}^{18}$. Of the $21 \%$ adolescents who ever used contraception, only $25 \%$ reported current use. Male condoms are the most widely used method of contraception. The majority of sexually active adolescents (95\%) ever used male 
condoms. Only 3\% and 1\% reported ever use of female condoms and injectables.

The results in this paper indicated that knowledge about traditional methods of contraception is very limited. The majority of respondents $(82 \%)$ did not know any natural method of contraception. Among the $12 \%$ who knew natural methods of contraception, most of them 79\% mentioned lactation amenorrhoea (breast feeding). None mentioned periodic abstinence. Knowledge of modern methods of contraception was universal (98\%) among adolescents. However, it should be noted that adolescents were more versed in male condoms (84\%) than female methods. Only $11 \%$, $2 \%$ and $3 \%$ of respondents knew about female pills, implant and injectables respectively. All adolescents expressed utter ignorance about both male and female sterilization methods.

It should be argued that limited contraceptive use was a result lack of knowledge about contraceptives among adolescents. Lack of knowledge has been fundamentally linked to absence of policy and irresponsibility of duty bearers. This negatively impacted on the acceptability and accessibility of contraceptives to adolescents. It should be noted that this dimension was not investigated in previous studies. Teachers argued that the absence of a NARH policy and National Schools' ARH syllabi provide obstacles in the teaching of ARH and more so contraceptive use. While as a substitute teachers in Mhondoro-Ngezi teach Guidance and Counseling $(\mathrm{G} \& \mathrm{C})$, it should equally be argued that it is of little importance to $\mathrm{RH}$ and contraceptive use among adolescents. It has been noted that G\&C does not have a national, provincial and district syllabi where schools can formulate their own syllabus. The study revealed that in G\&C lessons, teachers only teach lessons they want which range from dressing, cleanliness, how to study, covering books and punctuality. The fact that G\&C is not an examinable course worsens the time allocated to the subject since it is only given 40 minutes a week. In the end, the school set up rarely talks about $\mathrm{RH}$ and contraceptive use among adolescents. If ever taught as one teacher argued, they are bits and pieces of biology lessons. The study also revealed that teachers are not keen to talk and demonstrate condom use since they argued that it is contrary to societal expectations. In addition, teachers argued that one teacher from a neighboring school once distributed condoms to his school pupils and end up in police hands and subsequently charged by the Ministry of Education.

Lack of communication of $\mathrm{RH}$ education between adolescents and relevant stakeholders greatly creates a void in ARH information dissemination in Mhondoro-Ngezi. The study revealed that churches do not talk about contraception, sexual issues and worse still condom use and distribution among adolescents. The above mentioned factor is similar to what Mhloyi ${ }^{19}$ noted. Religious pastors argued that such lessons promotes early sexual debut, infidelity, increased rates of sexual activity and prostitution. They argued that the aforementioned activities are contrary to faithfulness and abstinence emphasized in the Bible. However, the International Conference of Population and Development (ICPD) paragraph 7:2 argued that once information is disseminated (even to adolescents);

..........informed individuals everywhere can and will act responsibly in the light of their own needs and those of their families and communities.

Moreover, some school-based control studies found that no evidence exists that provision of information to adolescents about contraception results in increased rates of sexual activity, earlier age of first intercourse, or a greater number of partners ${ }^{20,21,22}$. The studies noted that availability of contraceptives and contraceptive information to adolescents was not causally related to sexual experimentation.

Parents also argued that they cannot talk to their children about RH especially contraceptive use because of the (chinyarikani) register which exist between them and their children. They also argued that they will be perpetuating promiscuity and disrespect among adolescents. While parents argued that it is the responsibility of aunts, uncles and grandparents to provide IEC to adolescents, all the four FGDs concurred that there are no longer aunts nor uncles and grandparents to teach $\mathrm{RH}$ issues to adolescents due to migration, urbanization and the subsequent disruption of the extended family systems.

RH information dissemination and contraceptive use in particular, (in this study) is 
hampered by health service providers. HCP at Mhondoro-Ngezi are mandated and even do have key result areas which indicated that they hold outreach programmes in schools and other gatherings aimed at $\mathrm{RH}$ information dissemination to adolescents. However, it has been argued by both teachers and pupils that these health service providers never conducted any of such programs. In fact, they only see these nurses at sports days and political gatherings primarily providing first aid. In addition, while health service providers in Mhondoro-Ngezi stated that they have established a Youth Friendly Centre on 4 March 2007, it should be argued that the centre grounded down its functions on 2 June 2007 due to lack of facilities. Adolescents, worse still argued that the centre is too close to major clinic and are also worried about the old age of nurses who were supposed to mann the area.

It has been noted that in the absence of accurate RH information from duty bearers, adolescents rely on information from their illinformed peers. Resultantly, they are reluctant to use contraception because of the belief that condoms have holes which allow infection to pass through. There are also these beliefs that, contraceptive pills cause infertility, menstrual disorders and deformed babies.

Lack of knowledge by health care providers about the Reproductive Health Policy of 2003, the National Youth Policy of Zimbabwe of 2000 and the Reproductive Health Service Delivery Guidelines by $\mathrm{MOHCW}^{23}$ worsen the availability (especially female contraceptives) and accessibility of contraceptives to adolescents in Mhondoro-Ngezi. It should be noted that the afforested determinant was never revealed in previous studies. Results from the study revealed that whilst contraceptives are available at clinics in Mhondoro-Ngezi, healthcare providers hamper their accessibility. Interviews with health care providers in Mhondoro-Ngezi revealed that they do not offer contraception to unmarried adolescents below age 18 unless accompanied by parents. They argued that their practicing document forbid them to do so. However, it should be argued that such practice by health care providers displays lack of knowledge and malpractice at its zenith since the RH Policy ${ }^{24}$ Section 3.3.2 state that "Family planning services must be available and accessible to all those who need them regardless of age". In addition, the $\mathrm{MOHCW}^{22}$ (in its Reproductive Health Service Delivery Guidelines) Section 5.8 stated that:

Adolescents who have made an informed decision and choose to be sexually active should have an uninterrupted access to condoms and emergency contraception services regardless of age.

More so, the non-provision of contraception to unmarried adolescents by HCP due to lack of knowledge about existing policies is a violation of Section 4.4(vi) and Section 1.3.4 of the National Youth Policy in Zimbabwe which state that:

There is need to expand and create access to health services by young people regardless of age" and that "Everyone has the right to the enjoyment of the highest attainable standard of physical and mental health. Adolescents have the right to be informed about all matters relating to their daily lives including the right to $R H$ information and services.

Further-more it is a violation of the Convection of the Rights of the Child (Article 24) which states that "...state parties shall strive to ensure that no child is deprived of her or his right of access to health care services". More so, this is very contrary to the fundamental principles of the International Conference on Population and Development paragraph 7:2. The paragraph stipulated that:

The aim of family planning must be to enable couples and individuals to decide freely and responsibly the number and spacing of their children and to have the information and means to do so and to make informed choices and make available a full range of safe and effective methods.....

Whilst contraceptives are available at health centres and shops, old aged nurses (who seem to be always busy), judgmental and hostile attitudes and lack of privacy and confidentiality by shop keepers are deterrent factors to access and utilization. Adolescents argued that HCP have similar ages with their parents. As such the chinyarikani register will 
prevent them to ask any questions pertaining to contraceptive use. In addition, adolescents argued that they are failing to access contraceptives from clinics because of judgmental and hostility among nurses and shopkeepers who always think that any adolescent using contraceptives is a prostitute. Similar findings were echoed by $\mathrm{UNICEF}^{11}$, $\mathrm{UNFPA}^{25}$ and $\mathrm{UNFPA}^{26}$. Another study by UNFPA $^{26}$ revealed that, in Zimbabwe, 64 (16-26 years old) mystery clients visited public and private health facilities pretending to have experienced condom breakage the previous night and concerned about avoiding pregnancy. Surprisingly, only nine received emergency contraception prescription (three written by General Practitioners and six by pharmacists) while no treatment was prescribed for the remaining fifty-five because of the hostility of HCP.

The fact that nurses will disclose to other people about one's reproductive behavior is also a deterrent measure to accessibility of contraception to adolescents at health services.

While contraceptives are available at the clinics, it is the set up at the health service centres which are a bitter pill to swallow to adolescents. This has resultantly led to non-use of contraception by adolescents. The study revealed that condoms are placed on the recording desk, consultation room and treatment rooms. In most cases the aforementioned places will be full to capacity. The fact that the doors of the consultation and treatment rooms will be wide open, with nurses and other patients of different age groups pacing up and down, make adolescents feel uncomfortable to disclose their need of contraceptives. While condoms are available at clinics, shops and form community health distributors, it should be argued that they are not available in schools where about $80 \%$ of adolescents interact on daily basis.

The study also noted that the male condom is widely available and affordable to adolescents. Adolescents stated that male condoms cost one rand for a packet of three. However, the study also noted that whilst female contraceptives are available at clinics, (especially implants, pills, IUD and injectables), most adolescents are of school going ages. Resultantly, female adolescents cannot afford to pay the required US $\$ 20$ for the yearly card because they are economically dependent on parents and guardians. This has also contributed to non-use of contraceptives by adolescents.

It should also be argued that socio-cultural expectations have a part to play in noncontraceptive use among adolescents in MhondoroNgezi. The study also noted the double standards of culture when it comes to adolescent RH issues. In this study, society tends to condone premarital sex of adolescent females whilst men are left to graze around in order to display features of manhood and masculinity. Resultantly, male adolescents are more likely to access condoms than their female counterparts. The stigma of prostitution attached to female adolescents who use contraceptives greatly contribute to non-use of contraceptives. While culturally, society values virginity among adolescents (especially females), it should be argued that adolescents in Mhondoro-Ngezi are already sexually active. As a result, labeling them as prostitutes further perpetuates non-use of contraceptives. Society's attitude that contraceptives are only for married people leave adolescents with no option but fear of discovery of their sexual behavior and avoid seeking needed family planning contraception rather than risk punishment from parent.

\section{Conclusion}

In view of the factors determining contraceptive use to adolescents that have been discussed in this paper, it is imperative that the Ministry of Health and Child Welfare in Zimbabwe enact a National Adolescent Reproductive Health policy for the benefit of schools and healthcare providers' training. In addition, there is need for a multisectorial approach which includes the entire national participation of government, private sector, NonGovernmental Organizations, the family, religious institutions and traditional leaders, in working out interventions aimed at meeting the needs of adolescents. There is also need for the government, through the Ministry of Health and Child Welfare, to: establish Youth Friendly Centre manned by young healthcare providers; develop, distribute and disseminate information, education and communication materials to adolescents; and avail counseling services to adolescents. 


\section{Contribution of Authors}

Stanzia Moyo conceived the idea and drafted the topic and objectives of the study. She collected data. She jointly prepared the manuscript and analysed data with co-author. She unconditionally approved the manuscript.

Oswell Rusinga jointly prepared the manuscript and analysed data with corresponding author. $\mathrm{He}$ formatted the manuscript. He unconditionally approved the manuscript.

\section{References}

1. UNFPA. The State of World Population. New York: UNFPA, 2001.

2. Central Statistics Office [Zimbabwe]. National Profile Census Report. Harare: Government Zimbabwe, 2002.

3. Zimbabwe National Family Planning Council. Annual Report. Harare: Zimbabwe National Family Planning Council, 1999.

4. Cornejo MF, and Andina RB. Culturally appropriate information, education and communication strategies for improving adolescent reproductive health in Cusco, Peru, Population Council Frontiers in Reproductive Health CD-ROM, 2004.

5. Ministry of Health Child and Welfare. National HIV and AIDS Estimates. Harare: Government of Zimbabwe, 2009.

6. UNFPA. The State of World Population. New York: UNFPA, 2005.

7. White et al S.T. Raising Teens: A Synthesis of Research and a Foundation for Action. Boston: Centre for Health Communication, 2006.

8. Zimbabwe National Family Planning Council. Report on the National AIDS Control Review Programme. Harare: Zimbabwe National Family Planning Council, 1997.

9. Moyo S. Adolescent Reproductive Health Issues, A Case Study of Mhondoro-Ngezi. Unpublished Paper. Harare: Centre for Population Studies, University of Zimbabwe, 2008.

10. Dhliwayo E. Reproductive Health Issues of Adolescents at Mount Pleasant High School. Unpublished Paper.
Harare: Population Studies, University of Zimbabwe, 2009.

11. UNICEF. (2001). An Assessment of Adolescents Reproductive Health Needs in Zimbabwe. Harare: Training and Research Support Centre.

12. Ministry of Health Child and Welfare. Ministry of Health Return Form - Zimbabwe. Harare: Government of Zimbabwe, 2005.

13. Ministry of Health Child and Welfare. Ministry of Health Return Form - Zimbabwe. Harare: Government of Zimbabwe, 2006.

14. Gwanzura-Ottemoller FP. and Kesby MG. 'Let's talk about sex, baby...': Conversing with Zimbabwean children about HIV/AIDS. Children's Geographies 2005: 3 (2), 201-218.

15. Mhloyi, M. Family Sexuality and Reproductive Health in Zimbabwe. Harare: Weaver Press, 2003.

16. DiClemente, TN. and Wingood VW. Broadening the Horizon: Balancing Protection and Risks for Adolescents. London: Prentice Hall, 2003.

17. McFadden, P. Sex, Sexuality and the Problems of AIDS in Africa. In Meena, R. (Ed.), Gender in Southern Africa: Conceptual and Theoretical Issues (157-195). Harare: SAPES Trust, 1992.

18. Zimbabwe Demographic and Health Survey 2005-06

19. Mhloyi M. The Role of Church in HIV AND AIDS Intervention Strategies in Zimbabwe. Future Group International Zimbabwe Consultative Report, 1998.

20. Abin LS. et al. Evaluation of a Pregnancy Prevention Program for Urban Teenagers. Family Planning Perspective 1986; 18, 119-126.

21. Howard M. and Mitchell M. Preventing teenage pregnancy. Pediatrics Annual 1993; 22, 109-111.

22. Beach RK. Contraception for adolescents: Part 1. Adolescent Health Update 1994; 7:1.

23. Ministry of Health Child and Welfare. Reproductive Health Service Delivery Guidelines. Harare: Government of Zimbabwe, 2001.

24. Ministry of Health Child and Welfare. Reproductive Health Policy in Zimbabwe. Harare: Government of Zimbabwe, 2003.

25. UNFPA. A Rapid Assessment of the Reproductive Health Vulnerability of Adolescents, Youths and Women in Zimbabwe. New York: UNFPA, 2002.

26. UNFPA. State of the World Population: Investing in Adolescents Health and Rights. New York: UNFPA, 2003. 